\title{
Natural hazards for the Earth's civilization from space, 1. Cosmic ray influence on atmospheric processes
}

\author{
L. I. Dorman \\ Israel Cosmic Ray \& Space Weather Center and Emilio Segre' Observatory, affiliated to Tel Aviv University, Technion and \\ Israel Space Agency, Qazrin 12900, Israel \\ Cosmic Ray Department of IZMIRAN, Russian Academy of Science, Troitsk 142092, Moscow Region, Russia
}

Received: 4 July 2007 - Revised: 30 December 2007 - Accepted: 2 January 2008 - Published: 10 April 2008

\begin{abstract}
In this paper we give a short description of global natural disasters for the Earth's civilization from space: 1) Galactic and solar cosmic ray $(\mathrm{CR})$ influence on the atmospheric processes; 2) Impacts of great space magnetic storms during big Forbush-effects in CR, 3) Impacts of great radiation hazards from solar CR during flare energetic particle events, 4) Great impacts on planetary climate during periods of the Solar system capturing by molecular-dust clouds, 5) Catastrophic disasters from nearby Supernova explosions, and 6) Catastrophic disasters from asteroid impacts on the Earth. Some of these problems have been already studied (see e.g. Dorman, 1957, 1963a, b; Dorman and Miroshnichenko, 1968; Dorman, 1972, 1974, 1975a, b, 1978; Velinov et al., 1974; Miroshnichenko, 2001, 2003; Dorman, 2004, 2006, 2008). We present here a detailed treatment of the first disaster only, leaving to future papers the analysis of the other aspects.
\end{abstract}

\section{Introduction}

The main natural disasters for the Earth's civilization caused by space phenomena are the following:

Space natural disaster 1. Cosmic ray (CR) influence on the air ionization, nuclear and chemical reactions, on global atmospheric processes, on communication systems, on low cloud covering, and on global climate change. This type of space natural disaster will be considered in the present paper.

Space natural disaster 2. Global disaster from great space magnetic storms connected with big CR Forbushdecreases and their assessment by using the world-wide network of CR stations: great geomagnetic storms may affect adversely global technology systems, high frequency radio communications can be disrupted, electric power distribution

Correspondence to: L. I. Dorman

(lid@physics.technion.ac.il) grids blacked out when induced currents causes safety devices to trip, and atmospheric warming causes increased drag on satellites and anomalies in their operation. Moreover, increased frequency of myocardial infarction, brain stroke, car and train accidents have been observed during these events. Electric power and long oil tubes catastrophes have been observed in the past in Canada and other countries. By using on-line hourly CR data from the world-wide network of stations, it is possible to perform an accurate assessment of this natural hazard $15-20 \mathrm{~h}$ before the storm sudden commencement.

Space natural disaster 3. Global natural disaster from intense radiation hazards for astronauts, for crew and passengers on regular airline flights at the altitude of about $10 \mathrm{~km}$ ), and occasionally also for people on the ground level due to exceptional solar CR events. It was shown (References) that the predicted occurrence of such events (statistical distribution, examples from the past; we show that this advertisement, with high occurrence probability, can be given 30-60 min before the arrival of the more dangerous particle flux. This method is based on the well known fact that the main part of radiation hazard in space and in atmosphere is caused by particles with small energy (few hundreds $\mathrm{MeV}$ ) that reach the Earth $1-2 \mathrm{~h}$ after their acceleration on the Sun; on the contrary the relatively small flux of high-energy $(\geq 2 \mathrm{GeV}$ ) particles not involved in the radiation hazard, which can be detected by super neutron monitors, reach the Earth much more quickly. Several A few minutes of observation of the first-coming high-energy solar particles can give enough information on intensity, energy spectrum, transport parameters, and source function to make it possible to predict the time-space distribution of radiation hazard in interplanetary space (for astronauts and space-probe technology) and in the Earth atmosphere as a function of latitude (geomagnetic cut-off rigidity) and altitude.

Published by Copernicus Publications on behalf of the European Geosciences Union. 


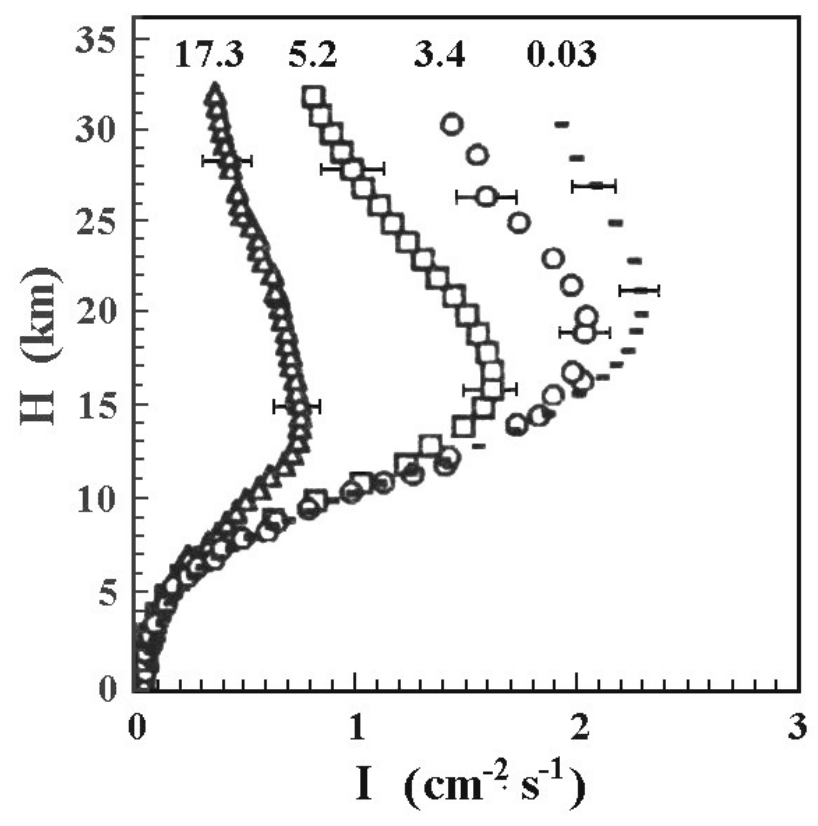

Fig. 1. The absorption curves of $C R$ in the atmosphere at different cutoff rigidities (numbers at the top in GV). The horizontal bars indicate the standard deviations (Ermakov et al., 1997).

Space natural disaster 4. Solar system captured by molecular-dust clouds. The prediction of the interaction of a dust-molecular cloud with the solar system can be performed by measurements of changes in the galactic CR distribution function. From the past we know that the dust from clouds between the Sun and the Earth leads to a decrease of solar irradiation flux leading to a sufficient decrease of global planetary temperature (by $5-7^{\circ}$ in comparison with $0.8^{\circ}$ from greenhouse effect). The plasma in a moving molecular dust cloud contains a frozen-in magnetic field; this field could modify the stationary galactic CR distribution outside the Heliosphere. The change in the distribution function can be significant, and it should be possible to identify these changes when the distance between the cloud and the Sun becomes comparable with the dimension of the cloud. The continuous observation of the time variation of CR distribution function for many years should provide the possibility of determining the direction and the speed of the cloud relative to the Sun, as well as its geometry. Therefore, one could predict its evolution in space and determine whether it will catch the Sun or not. In the case of high probability of capture, we could predict the time of the capture and how long the solar system will be inside the cloud.

Space natural disaster 5. Catastrophic disasters from nearby Supernova explosions. Prediction of the radiation hazard produced by CR particles generated in a nearby Supernova explosion (SE). From the energetic balance of $\mathrm{CR}$ in the Galaxy it was estimated that the full power for $\mathrm{CR}$ production is $\mathrm{W}_{\mathrm{CR}} \sim 3 \times 10^{40} \mathrm{erg} / \mathrm{s}$. Now it is commonly ac-

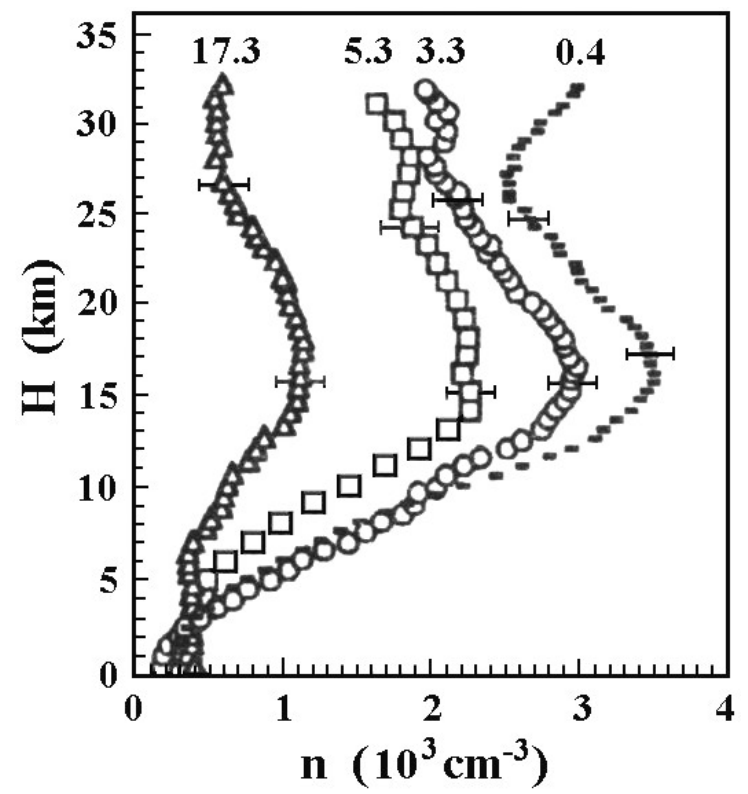

Fig. 2. The ion concentration profiles as a function of altitude for different geomagnetic cutoff rigidities (numbers at the top in GV). The horizontal bars indicate the standard deviations (Ermakov et al., 1997).

cepted that the Supernova explosions are the main source of galactic CR. At each explosion the average energy transferred to $\mathrm{CR}$ is $\mathrm{E}_{\mathrm{SE}} \sim 10^{50}-10^{51} \mathrm{erg}$. From this quantity we can determine the expected frequency of SE in our Galaxy and in vicinity of the Sun and estimate the probability of Supernova explosions at different distances from the Sun, the expected radiation hazard, and its variation with time. It was shown that in some cases the level of radiation may increase by about 1000 times in comparison with present level, reaching levels extremely dangerous for the Earth's civilization and biosphere. By high energy CR measurements by ground and underground muon telescopes and low-latitude neutron monitors on the Earth it is possible to obtain information on the source function and diffusion coefficient many years before the real radiation hazard will involve the Earth. On the basis of this information it is possible to perform an accurate forecasting on the time developing of the radiation hazard in space and in the atmosphere at different altitudes and cutoff rigidities (different geomagnetic latitudes), by using the method of coupling functions. On the basis of the obtained forecast the Earth's civilization will have enough time (at least several tens of years) to prepare an alternative life underground and in special protected buildings, where to live for several hundreds years.

Space natural disaster 6. Catastrophic disasters from asteroid impacts on the Earth. These catastrophic disasters occurred several times in the past. At present, by modern methods of Astronomy, the trajectory of dangerous asteroids can be exactly determined. By the impact between the asteroid 


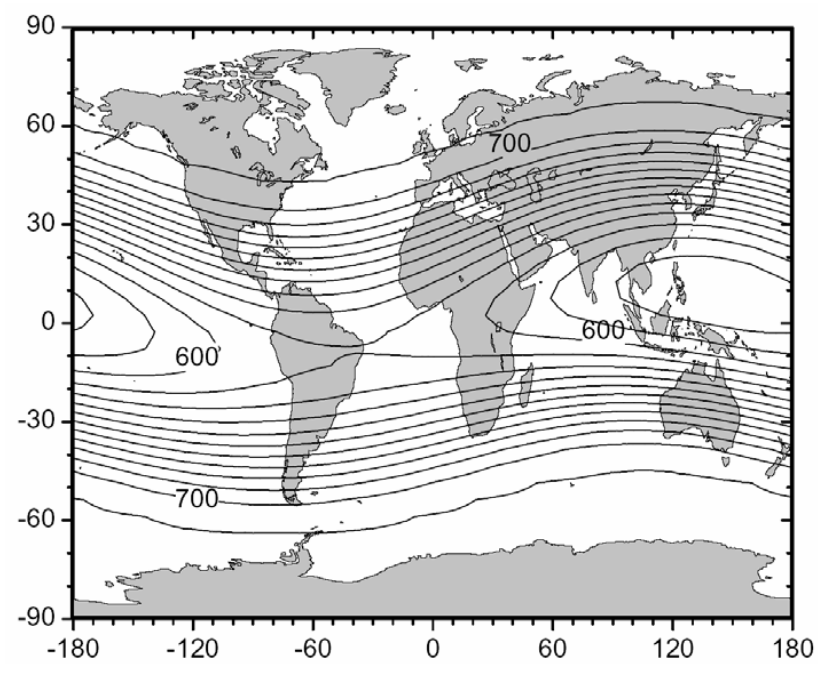

Fig. 3. Calculated equilibrium galactic $C R$ induced ionization at the altitude of $3 \mathrm{~km}\left(h=725 \mathrm{~g} / \mathrm{cm}^{2}\right)$ for the year 2000 . Contour lines are given as the number of ion pairs per $\mathrm{cm}^{3}$ in steps of $10 \mathrm{~cm}^{-3}$ (Usoskin et al., 2004).

and a particularly powerful rocket, the trajectory of the asteroid could be slightly changed avoiding a catastrophic interaction.

2 CR influence on the air ionization, nuclear and chemical reactions, on radio wave propagation, on atmospheric electrical effects and thunderstorms, on low cloud covering and on global climate change

\subsection{Air ionization by galactic CR}

Figure 1 shows experimental data of Ermakov et al. (1997) on galactic CR generation of secondary particles and absorption for different cutoff rigidities, and Fig. 2 - total ionization of atmosphere by galactic CR (primary and secondary) in dependence of altitude.

The planetary distribution of ionization at the altitude of $3 \mathrm{~km}$, calculated by Usoskin et al. (2004), is shown in Fig. 3 for the year 2000, and its time variation during 1950-2000 is presented in Fig. 4.

2.2 Air ionization by solar CR and influence on radio-wave communications

During great solar energetic particle events the air ionization increased by many times, leading to a sufficient influence on radio-wave communications (see Dorman, 1963a, b; Velinov et al., 1974; Dorman, 2004). Especially great disruptions in short radio wave communications were observed during great solar CR event at 23 February 1956. In Fig. 5 we show how the air ionization was changing during some big solar CR events in the period 1989-2001, computed by Quack et

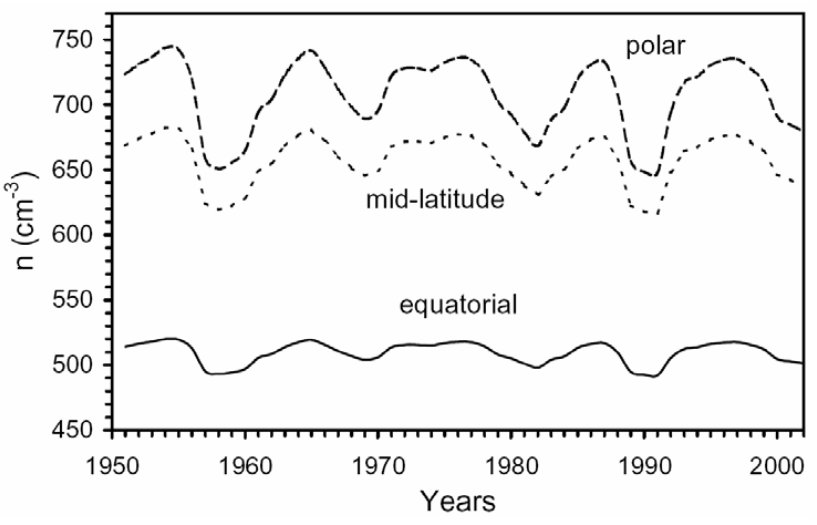

Fig. 4. Calculated time profiles of the annual ionization at altitude of $3 \mathrm{~km}\left(h=725 \mathrm{~g} / \mathrm{cm}^{2}\right)$, induced by galactic CR, for three regions: polar $\left(R_{c}<1 \mathrm{GV}\right)$, mid-latitudes $\left(R_{c} \approx 6 \mathrm{GV}\right)$ and equatorial ( $\left.R_{C} \approx 15 \mathrm{GV}\right)$ regions. According to Usoskin et al. (2004).

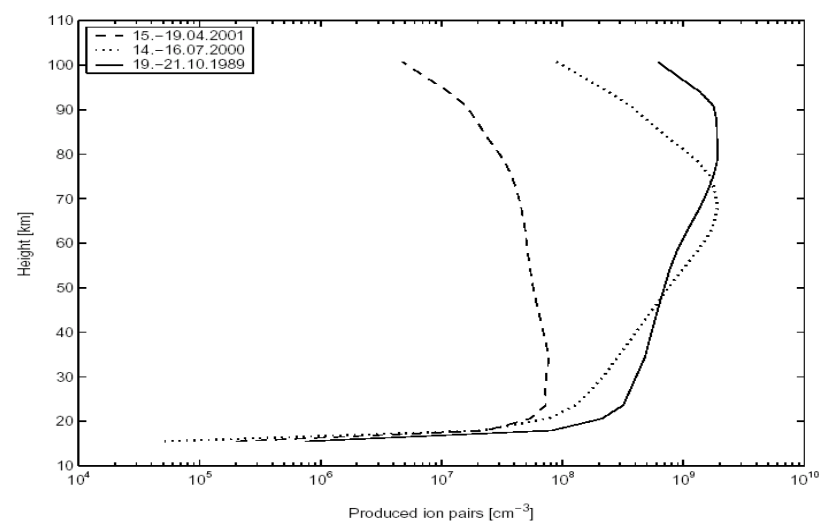

Fig. 5. Total ionization during big solar CR events in October 1989, July 2000, and April 2001 (Quack et al., 2001).

al. (2001) on the basis of data on observed energy spectra of solar CR.

2.3 Galactic and solar CR influence on atmospheric chemical processes

This problem was considered in detail by Dorman (2004). We show in Fig. 6, as an example, the production of nitrates in the Earth's atmosphere by galactic and solar CR, and in Fig. 7 the reduction of ozone contents during some solar CR events.

\subsection{Galactic and solar CR influence on atmospheric nuclear processes}

As result of nuclear reactions of galactic and solar CR in the Earth's atmosphere a lot of cosmogenic stable and radioactive isotopes $\left({ }^{3} \mathrm{H},{ }^{3} \mathrm{He},{ }^{7} \mathrm{Be},{ }^{10} \mathrm{Be},{ }^{14} \mathrm{C}\right.$, and others) are produced. The measurements of ${ }^{10} \mathrm{Be}$ contents in ice columns 


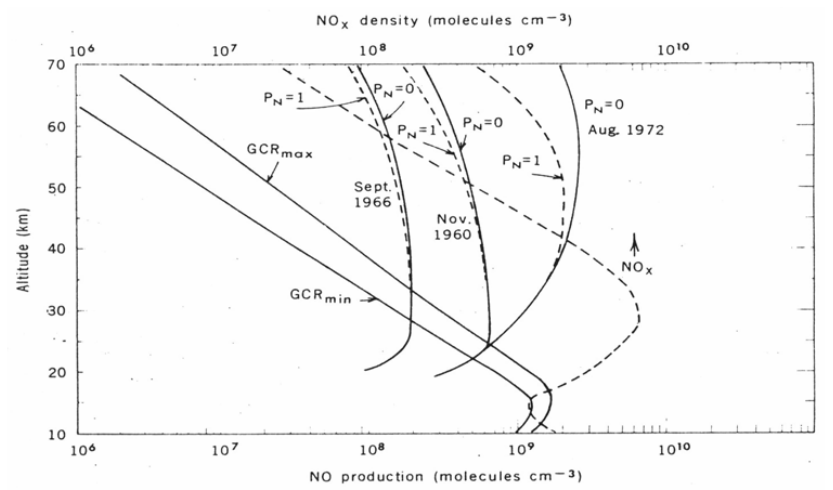

Fig. 6. Nitrates production by galactic CR during minimum and maximum of solar activity (correspondingly GCR $\max$ (NOT VISIBLE) and $\mathrm{GCR}_{\min }$ ) and during some solar CR events in November 1960, September 1966, and August 1972 (Crutzen et al., 1975).

from Antarctica and Greenland are widely used for determining galactic CR intensity and solar CR events in the past. The content of ${ }^{14} \mathrm{C}$ in annual wood rings is utilized for determining exchange processes between atmosphere, biosphere, and oceans, as well as CR intensity in past and for dating (wellknown radiocarbon method). It is important that by cosmogenic isotopes it is possible to find out catastrophic events in the Earth's environment in the past (see Dorman, 2004).

2.5 CR influence on atmospheric electric field and thunderstorms, global Earth's charge and global electric current

The influence of CR on thunderstorms and atmospheric electric field was intensively investigated in the frame of kinetic theory of discharges by Gurevich et al., 1992, 1999, 2001; Gurevich and Milikh, 1999; Gurevich and Zybin, 2001. It was shown that discharges are not possible without the presence of $\mathrm{CR}$ in the observed atmospheric electric fields. Only owed to the secondary CR relativistic electrons, which in the atmospheric electric field create runaway electron avalanches, thunderstorm discharges become possible. On the other hand, also Ermakov (1992), Ermakov and Stozhkov (1999, 2003) connected thunderstorms discharges with CR, but they assumed that the main cause are the atmospheric showers of energetic particles generated by primary CR with energy $\geq 10^{14} \mathrm{eV}$ (the frequency of their generation in a cloud with a dimension of several $\mathrm{km}$ is about 1000/s). It is well known that the Earth has about constant negative charge $Q \approx 6 \times 10^{5} \mathrm{C}$. As a result, in quiet conditions there are electric fields of about $-130 \mathrm{~V} / \mathrm{m}$ directed to the Earth's surface. Between the Earth's surface and the neutral layer in ionosphere at the altitudes of $55-80 \mathrm{~km}$ there is a flow of electric current with average density $J \approx 10^{-12} \mathrm{~A} / \mathrm{m}^{2}$. The total discharged current in all atmosphere is about 1800 $\mathrm{A}$, and this current may discharge the negative charge of the

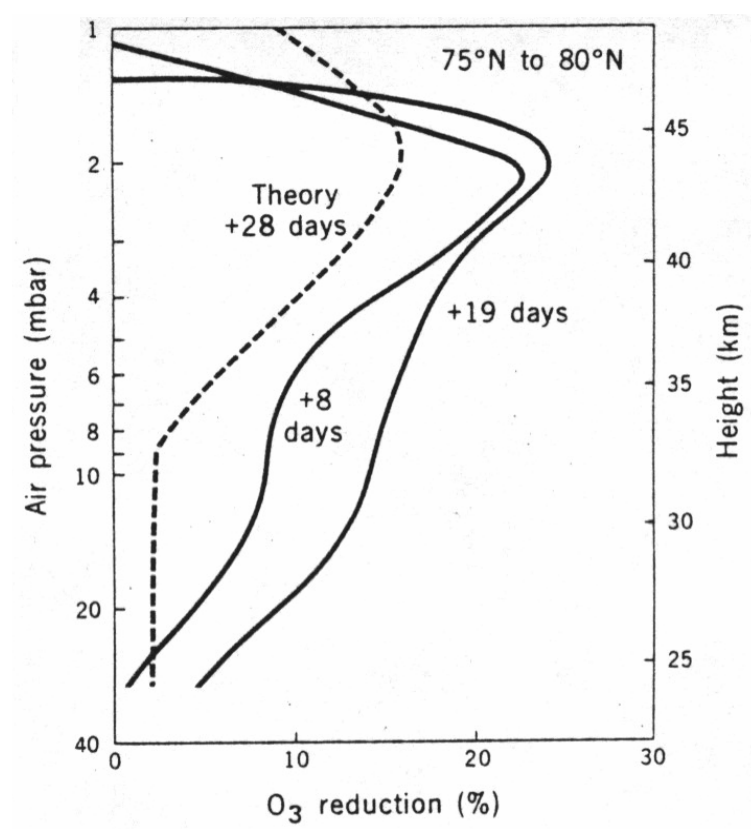

Fig. 7. Percentage decrease of the $\mathrm{O}_{3}$ partial pressure versus air pressure derived from the average of the 7 days before 4 August 1972 and 7 day periods centered on 8 and 19 days after the solar CR event (solid lines). According to Heath et al. (1977).

Earth in 5-6 min (Chalmers, 1967). This discharge is indeed compensated by many thousands thunderstorms and electron precipitations which are charging the Earth negatively. A global electric circuit in the low atmosphere is provided by $\mathrm{CR}$ which, through the ionization of air atoms, assure air conductivity. Therefore, CR may play an important key role in keeping about constant the Earth's global negative charge (see Dorman, 2004).

2.6 CR influence on planetary cloud-covering and longterm climate change

In Fig. 8 we show results of Marsh and Swensmark (2000a, b) on the comparison between $\mathrm{CR}$ intensity variation and variations in planetary cloud coverage.

Figure 8 shows that a very good correlation is found for the low clouds. This good correlation can be explained by considering the ionization of air by CR. The decreasing of CR intensity leads to decreasing of the air ionization, causing a decreasing of planetary clouding coverage, and a corresponding increasing of planetary ground air temperature.

In Fig. 9 it is shown another example of the influence of space effects on the Earth's climate: variation of air surface temperature during Maunder minimum in connection with variation in ${ }^{10} \mathrm{Be}$ concentration (which is proportional to $\mathrm{CR}$ intensity).

From Fig. 9 can be seen that again the main cause of the long-term changes in climate is time variation of CR inten- 


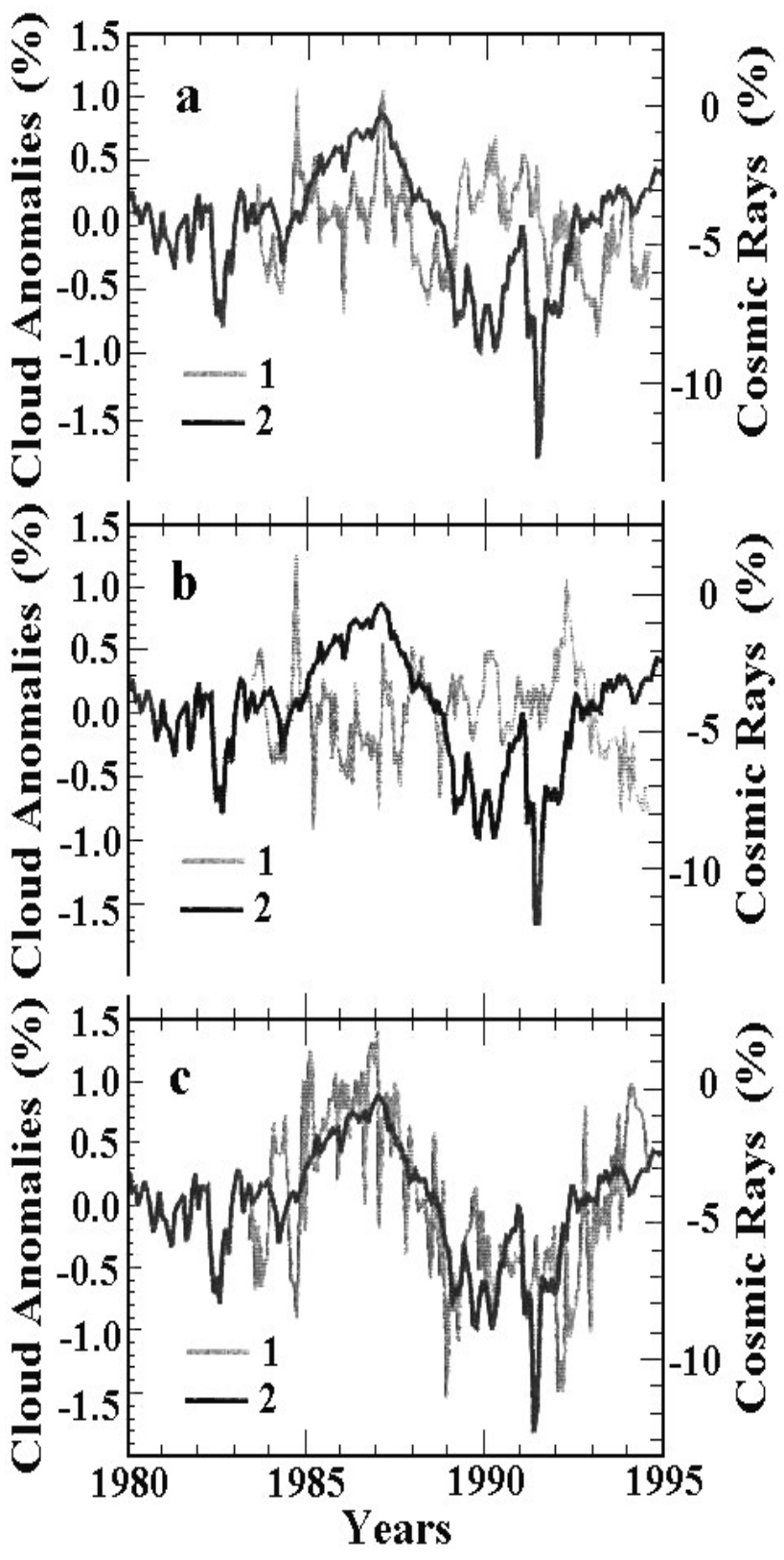

Fig. 8. CR intensity according to Huancayo/Haleakala NM (cut off rigidity $12.9 \mathrm{GV}$, normalized to October 1965 , curve 2) in comparison with global average of monthly cloud coverage anomalies (curve 1) for: (a) - high clouds, $\mathrm{H}>6.5 \mathrm{~km}$, (b) - middle clouds, $6.5 \mathrm{~km}>\mathrm{H}>3.2 \mathrm{~km}$, and (c) - low clouds, $\mathrm{H}<3.2 \mathrm{~km}$. According to Marsh and Swensmark (2000a, b).

sity, and with increasing of CR intensity increases air ionization, which leads to increasing of low clouds covering and corresponding decreasing of the air surface temperature. In papers Dorman (2005), Belov et al. (2005) were developed methods of forecasting the expected CR intensity long-term variation and determining the part of global climate change, caused by CR influence on the planetary low clouds covering (from few years up to 11 years ahead).
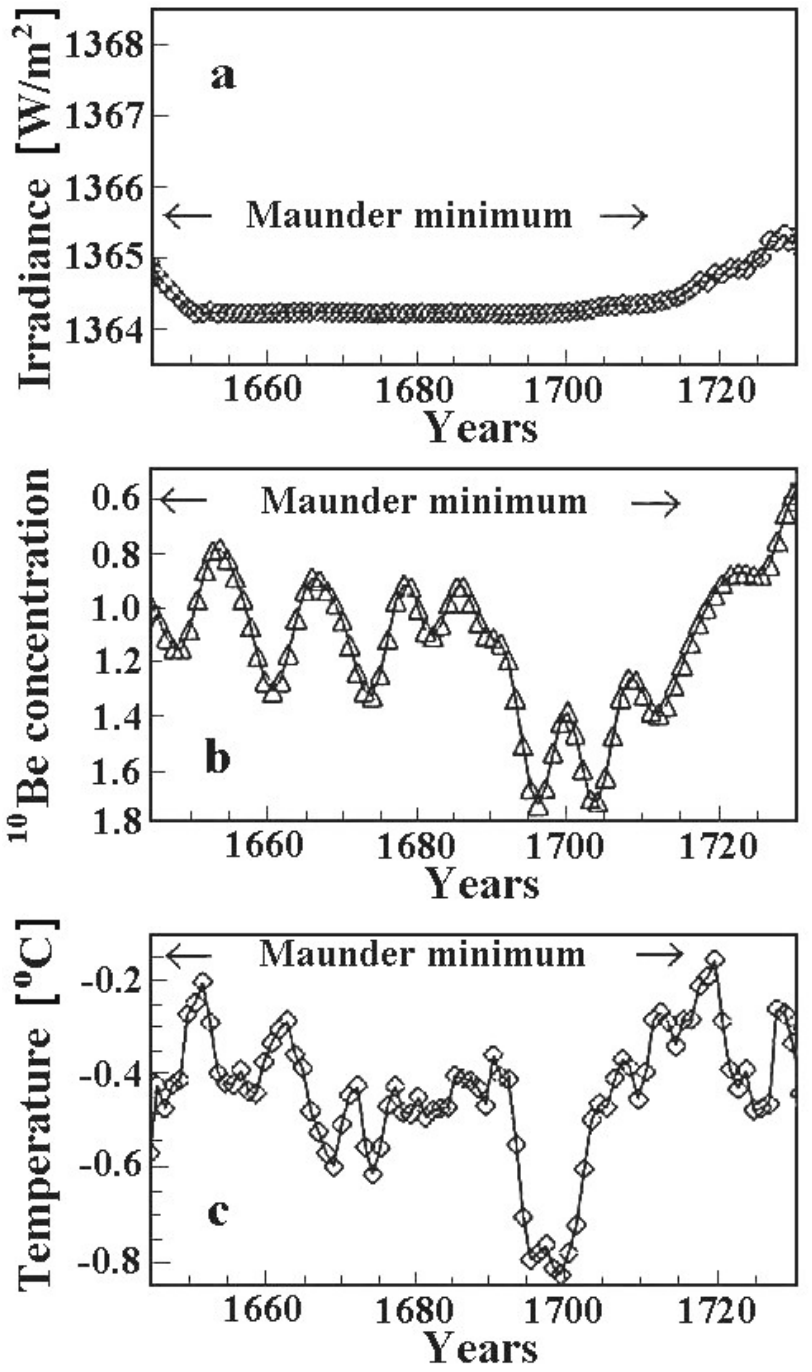

Fig. 9. Situation in the Maunder minimum: (a) variation in reconstructed solar irradiance from Lean et al. (1995); (b) variation in ${ }^{10} \mathrm{Be}$ concentration from Beer et al. (1991); (c) reconstructed air surface temperature for the northern hemisphere from Jones et al. (1998). According to Swensmark (2000).

\section{Conclusions}

We presented in this paper a short description of the main space natural disasters for the Earth's civilization. we considered in more detail only one possible disaster, connected with space phenomena influence on atmospheric processes: influence of galactic and solar CR on ionization, nuclear and chemical reactions, on atmospheric electrical processes, on planetary cloudiness and global climate changes.

Edited by: P. Lagos

Reviewed by: A. Chilingarian and H. Mavromichalaki 


\section{References}

Beer, J., Raisbeck, G. M., and Yiou, F.: Time variations of ${ }^{10} \mathrm{Be}$ and solar activity, in: The Sun in Time, edited by: Sonett, C. P., Giampapa, M. S., and Matthews, M. S., University of Arizona Press, 343-359, 1991.

Belov, A. V., Dorman, L. I., Gushchina, R. T., Obridko, V. N., Shelting, B. D., and Yanke, V. G.: Prediction of expected global climate change by forecasting of galactic cosmic ray intensity time variation in near future based on solar magnetic field data, Adv. Space Res., 35, 491-495, 2005.

Chalmers, J. A.: Atmospheric Electricity, Pergamon Press, 1967.

Crutzen, P. J., Isaksen, I. S. A., and Reid, G. C.: Solar proton events: stratospheric sources of nitric oxide, Science, 18, 4201, 457-459 (1975).

Dorman, L. I.: Cosmic Ray Variations, Gostekhteorizdat, Moscow, US Department of Defense, Ohio Air-Force Base, 495 pp., 1957 (english translation: 1958).

Dorman, L. I.: Cosmic Ray Variations and Space Research, Nauka, Moscow, 1023 pp., 1963a.

Dorman, L. I.: Geophysical and Astrophysical Aspects of Cosmic Rays, in: Progress in Physics of Cosmic Ray and Elementary Particles, edited by: Wilson, J. G. and Wouthuysen, S. A., Vol. 7, North-Holland Publ. Co., Amsterdam, 320 pp., $1963 b$.

Dorman, L. I.: Meteorological Effects of Cosmic Rays, Nauka, Moscow, 1972.

Dorman, L. I.: Cosmic Rays: Variations and Space Exploration, North-Holland Publ. Co., Amsterdam, 1974.

Dorman, L. I.: Variations of Galactic Cosmic Rays, Moscow State University Press, Moscow, 214 pp., 1975a.

Dorman, L. I.: Experimental and Theoretical Principles of Cosmic Ray Astrophysics, Fizmatgiz, Moscow, 1975b.

Dorman, L. I.: Cosmic Rays of Solar Origin, in: Summary of Science, Space Investigations, Vol. 12, VINITI, Moscow 1978.

Dorman, L. I.: Cosmic Rays in the Earth's Atmosphere and Underground, Kluwer Academic Publishers, Dordrecht/Boston/London, p. 32+855, 2004.

Dorman, L. I.: Estimation of long-term cosmic ray intensity variation in near future and prediction of their contribution in expected global climate change, Adv. Space Res., 35, 496-503, 2005.

Dorman, L. I.: Cosmic Ray Interactions, Propagation, and Acceleration in Space Plasmas, Springer, Netherlands, p. 31+842, 2006.

Dorman, L. I.: Cosmic Rays in Magnetospheres of the Earth and Other Planets, Springer, Netherlands, in press, 2008.

Dorman, L. I. and Miroshnichenko L. I.: Solar Cosmic Rays, Fizmatgiz, Moscow, 508 pp., 1968 (English translation published for NASA and NSF in 1976 (TT 70-57262/NASA TT F-624), Washington, D.C.).

Ermakov, V. I.: Lightning iniciation by galactic cosmic rays, Proc. 9th Intern. Conf. Atmosper. Electr., St. Petersburg, 2, 485-488, 1992.
Ermakov, V. I. and Stozhkov, Y. I.: New mechanism of thundercloud and lightning production, Proc. 11th Intern. Conf. Atmosper. Electr., Alabama, USA, 242-245, 1999.

Ermakov, V. I. and Stozhkov, Y. I.: Cosmic rays in the mechanism of thundercloud production, Proc. 28th Intern. Cosmic Ray Conf., Tsukuba, 7, 4157-4160, 2003.

Ermakov, V. I., Bazilevskaya, G. A., Pokrevsky, P. E., and Stozhkov, Yu. I.: Cosmic rays and ion production in the atmosphere, Proc. 25th Intern. Cosmic Ray Conf., Durbin, 7, 317-320, 1997.

Gurevich, A. V., Carlson, H. C., Medvedev, Yu. V., and Zybin, K. P.: Kinetic theory of runaway breakdown in inhomogeneous and thundercloud electric field, Phys. Lett., A282, 180-185, 2001.

Gurevich, A. V. and Milikh, G. M.: Generation of X-rays due to multiple runaway breackdown inside thunderclouds, Phys. Lett., A262, 457-463, 1999.

Gurevich, A. V. and Zybin, K. P.: Runaway breakdown and electric discharges in thunderstorms, UFN, 171, 11, 1177-1199, 2001.

Gurevich, A. V., Zybin, K. P., and Russel-Dupre, R.: Runaway electron mechanism of air breakdown and preconditioning during thunderstorms, Phys. Lett., A165, 463-468, 1992.

Gurevich, A. V., Zybin, K. P., and Russel-Dupre, R. A.: Lightning initiation by simultaneous effect of runaway breakdown and cosmic ray showers, Phys. Lett., A254, 79-87, 1999.

Heath, D. F., Krüger, A. J., and Crutzen, P. J.: Solar proton event: influence on stratospheric ozone, Science, 197, 886-889, 1977.

Jones, P. D., Briffa, K. R., Barnett, T. P., and Tett, S. F. B.: High resolution paleoclimatic records for the last millennium: interpretation, integration and comparison with general circulation model control run temperatures, The Holocene, 8, 455-471, 1998.

Lean, J., Beer, J., and Breadley, R.: Reconstruction of solar irradiance since 1610: implications for climate change, Geophys. Res. Lett., 22, 3195-3198, 1995.

Marsh, N. D. and Swensmark, H.: Low cloud properties influenced by cosmic rays, Phys. Rev. Lett., 85, 5004-5007, 2000a.

Marsh, N. and Swensmark, H.: Cosmic rays, clouds, and climate, Space Sci. Rev., 94, 1-2, 215-230, 2000b.

Miroshnichenko, L. I.: Solar Cosmic Rays, Kluwer Academic Publishers, Dordrecht/Boston/London, 2001.

Miroshnichenko, L. I.: Radiation Hazard in Space, Kluwer Academic Publishers, Dordrecht/Boston/London, 2003.

Quack, M., Kallenrode, M. B., König, M., et al.: Ground level events and consequences for stratospheric chemistry, Proc. 27th Intern. Cosmic Ray Conf., Hamburg, 10, 4023-4026, 2001.

Swensmark, H.: Cosmic rays and Earth's climate, Space Sci. Rev., 93, 175-185, 2000.

Usoskin, I. G., Gladysheva, O. G., and Kovaltsov, G. A.: Cosmic ray induced ionization in the atmosphere: spatial and temporal changes, J. Atmos. Solar-Terr. Phys., 66, 18, 1791-1796, 2004.

Velinov, P., Nestorov, G., and Dorman, L.: Cosmic Ray Influence on Ionosphere and Radio Wave Propagation, Bulgaria Academy of Sciences Press, Sofia, 1974. 